\title{
Meckel-Gruber Syndrome: A Case Report at RHUH
}

\author{
Darido Jessie $^{1,2}$, Khazaal Janoub ${ }^{1,2}$, El Hachem Larissa ${ }^{3}$, Luk Melodie ${ }^{4}$, Chahine Rabih ${ }^{1}$ \\ ${ }^{1}$ Department of Obstetrics and Gynecology, Rafic Hariri University Hospital, Beirut, Lebanon \\ ${ }^{2}$ Department of Obstetrics and Gynecology, Faculty of Medical Sciences, The Lebanese University, Beirut, Lebanon \\ ${ }^{3}$ Department of Obstetrics and Gynecology, Faculty of Medicine, Holy Spirit University of Kaslik, Kaslik, Lebanon \\ ${ }^{4}$ Department of Obstetrics and Gynecology, Faculty of Medicine, Paris 12 University, Creteil, Ile de France, France
}

\section{Email address:}

jesydarido@hotmail.com (D. Jessie)

\section{To cite this article:}

Darido Jessie, Khazaal Janoub, El Hachem Larissa, Luk Melodie, Chahine Rabih. Meckel-Gruber Syndrome: A Case Report at RHUH. Journal of Gynecology and Obstetrics. Vol. 8, No. 4, 2020, pp. 108-112. doi: 10.11648/j.jgo.20200804.17

Received: July 1, 2020; Accepted: July 13, 2020; Published: August 10, 2020

\begin{abstract}
A rare lethal autosomal recessive condition called Meckel-Gruber Syndrome (MGS) has a clinical and genetical heterogenicity. A 15-year-old primigravid presented at the $16^{\text {th }}$ gestational week of her pregnancy at the Rafic Hariri University Hospital (RHUH) for antenatal ultrasonography. She had a third degree consanguineous marriage with no previous uterine scars, no previous invasive testing, no previous fetal anomaly, no comorbidities and no family history of congenital anomalies. On the other hand, her current pregnancy was not the result of an Artificial Reproductive technique (ART) and her medication at home was only based on vitamins and calcium. The antenatal ultrasonography showed the following features: (1) occipital encephalocele (2) diffuse subcutaneous edema (3) bilateral dysplastic kidneys and the bladder was not visualized. These features were suggestive of Meckel Gruber Syndrome (MGS). We concluded that ultrasound in the context of genetic diseases, especially our MGS case, is considered as a secondary prevention tool.
\end{abstract}

Keywords: Meckel-Gruber, Syndromes, Ultrasound, Antenatal Diagnosis, Congenital Abnormalities

\section{Introduction}

Johann Friedrich Meckel was the first author to report this syndrome in 1822. Other cases were also reported by G. B. Gruber in 1934. Those cases were reported were not diagnosed antenatally especially that the era of ultrasound hadn't started yet at that time [1-4]. Epidemiologically, the incidence of MGS is not very precisely known. As mentioned by some authors, it is almost around 1:140 000 [5]. More than 200 cases of MGS were reported in the literature.

We hereby report one of rare lethal autosomal recessive conditions called Meckel-Gruber Syndrome (MGS) also known as dysencephalia splanchnocystica with its clinical and genetical heterogenicity.

\section{Case Presentation}

A 15-year-old primigravid (G1 P0 A0 L0 D0 E0) Syrian lady presented at the $16^{\text {th }}$ gestational week of her pregnancy at the Rafic Hariri University Hospital (RHUH) for antenatal ultrasonography. She was referred by the outpatient department (OPD) of the hospital after her first visit as a routine measurement with no evident clinical abnormal findings.

She had a third degree consanguineous marriage with no previous uterine scars, no previous invasive testing, no previous fetal anomaly, no comorbidities and no family history of congenital anomalies. On the other hand, her current pregnancy was not the result of an Artificial Reproductive technique (ART) and her medication at home was only based on vitamins and calcium.

The Ultrasound examination was performed in a relaxed and informal atmosphere, the woman was in a supine position, the operator on her right side, holding the probe with her right hand and at the right height relative to the woman's abdomen and the ultrasound machine positioned to the left of the examination couch.

The antenatal ultrasonography showed a single intrauterine pregnancy of variable presentation and a posterior marginal placenta. The measurements were commensurate with 16 weeks +1 with an Estimated Fetal Weight of 175 grams. The Fetal Heart Rate was normal. It also revealed the following 
features: (1) occipital encephalocele (2) diffuse subcutaneous edema (3) bilateral dysplastic kidneys and the bladder was not visualized. These features were suggestive of Meckel Gruber Syndrome (MGS).

The patient was counseled regarding the lethal outcome of this syndrome and about the ultrasound limitations and possibility of missed additional anomalies.

We report hereby the ultrasound images that were saved:

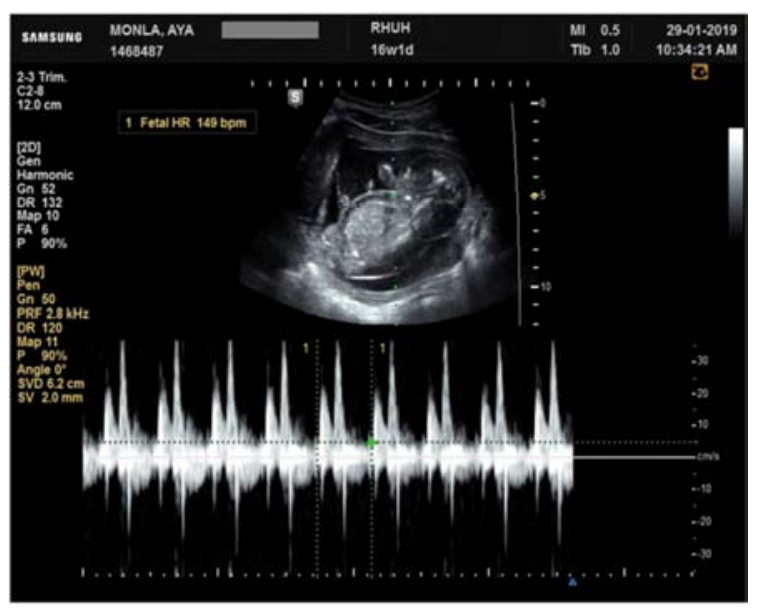

Figure 1. Positive Fetal Heart Detection.

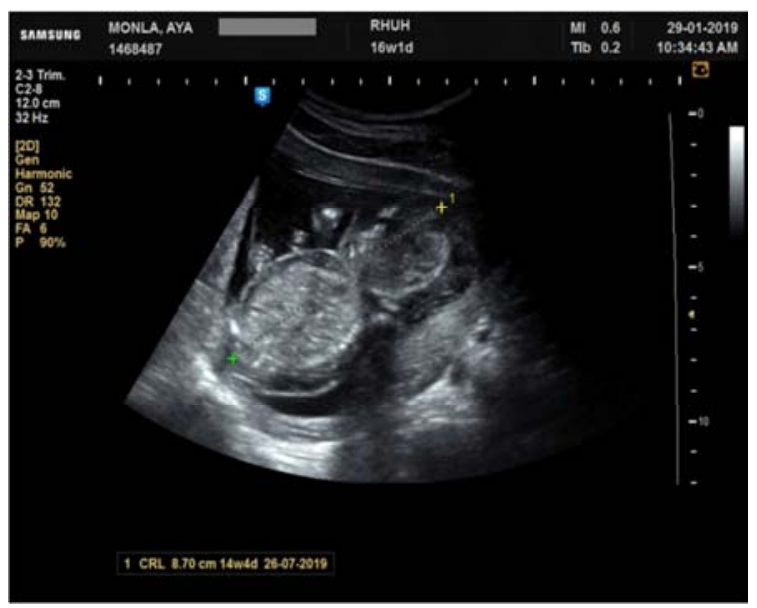

Figure 2. End-Points identification and CRL measurement.

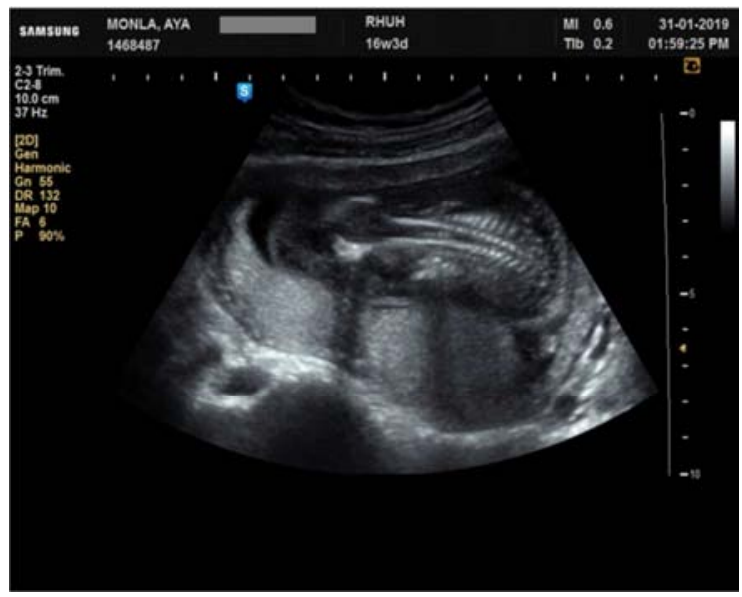

Figure 3. Longitudinal section of the Fetal Spine.

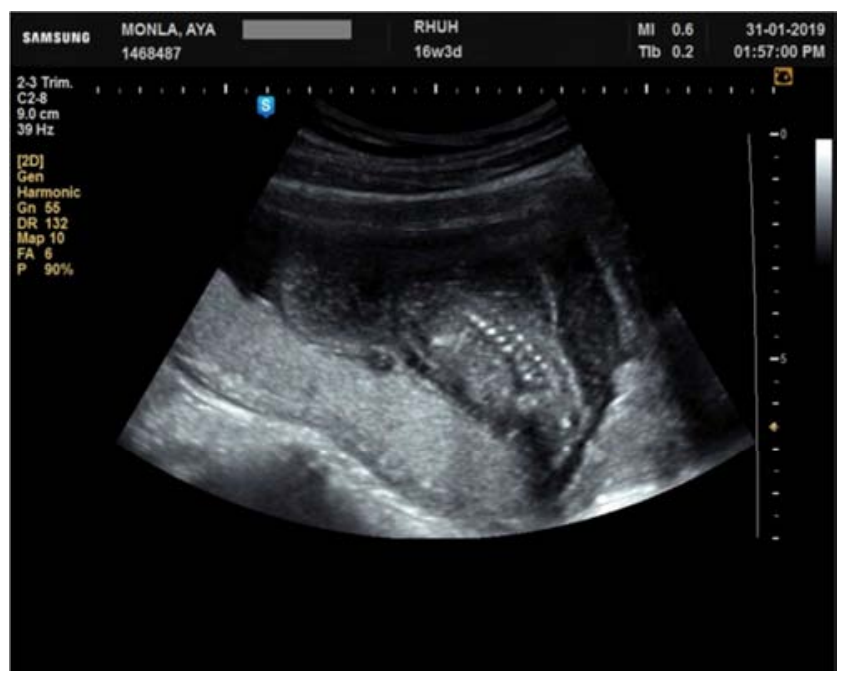

Figure 4. Coronal section of the Fetal Spine.

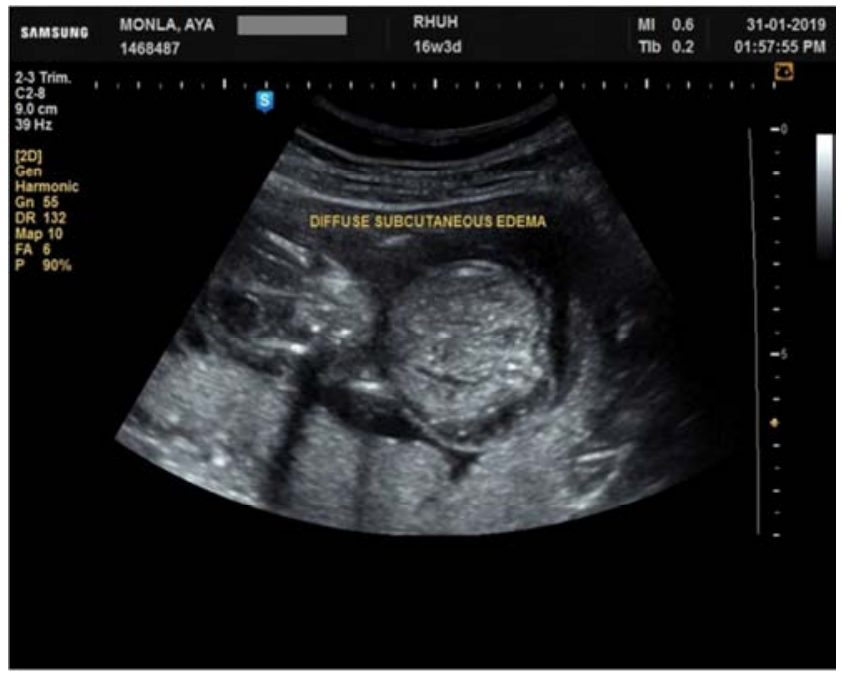

Figure 5. Coronal section of fetal body demonstrating diffused subcutaneous edema.

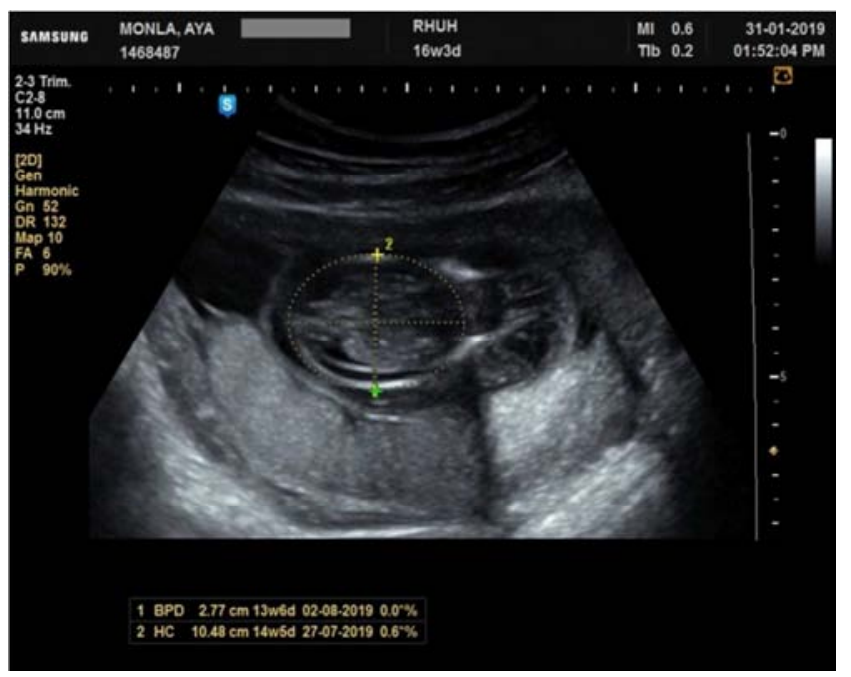

Figure 6. "Outer to Outer" Bi Parietal Diameter and Head Circumference measurements - Transverse section of the fetal head, calipers placed on the outer borders of parietal and distal parietal bones. 


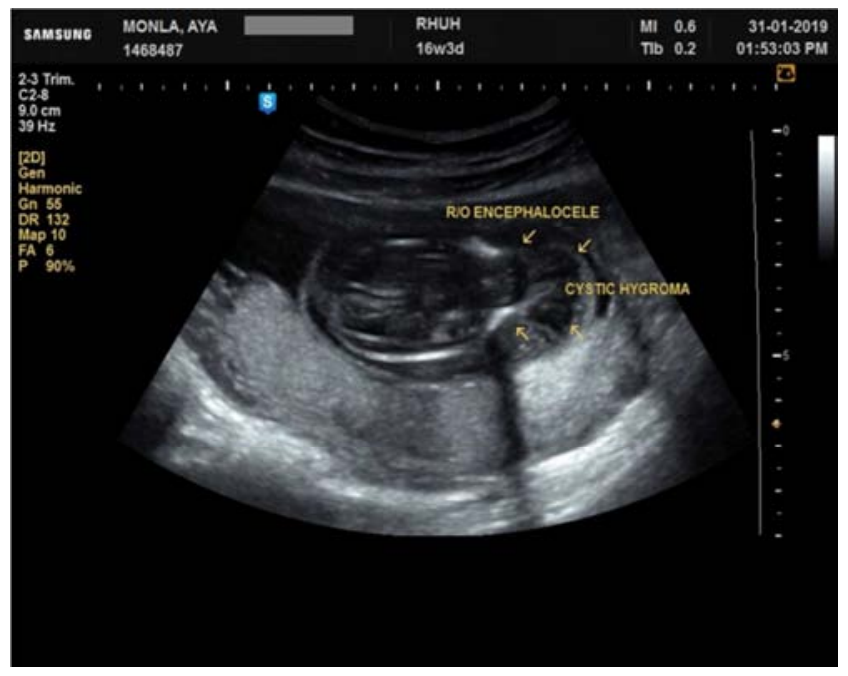

Figure 7. Axial view of the fetal head showing Occipital Encephalocele (arrows) with an open cranial defect and a protruding complex mass that includes brain tissue.

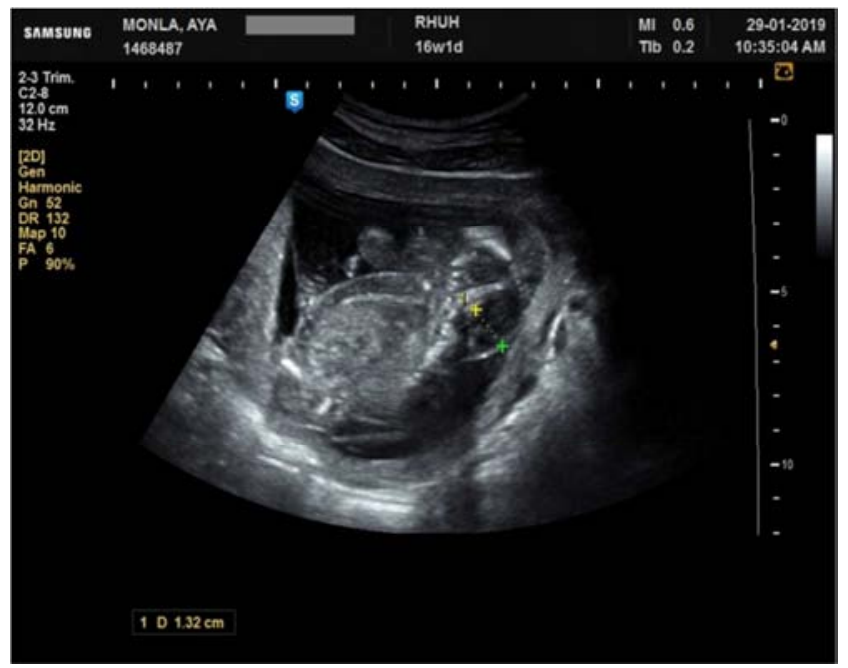

Figure 8. Sagittal image of the fetus showing occipital encephalocele (cursors).

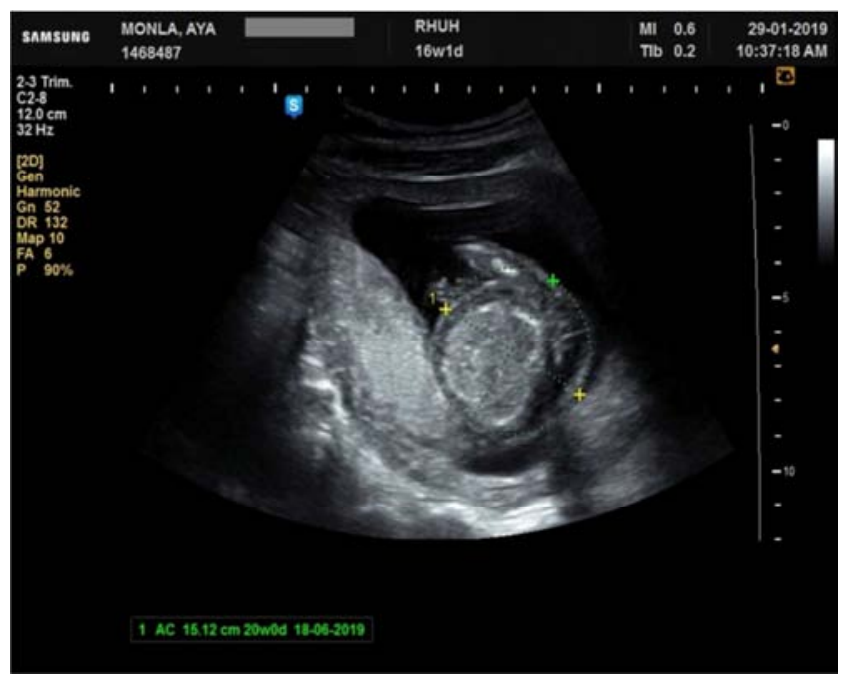

Figure 9. Measurement of the abdominal circumference on a transverse section of the fetal abdomen.

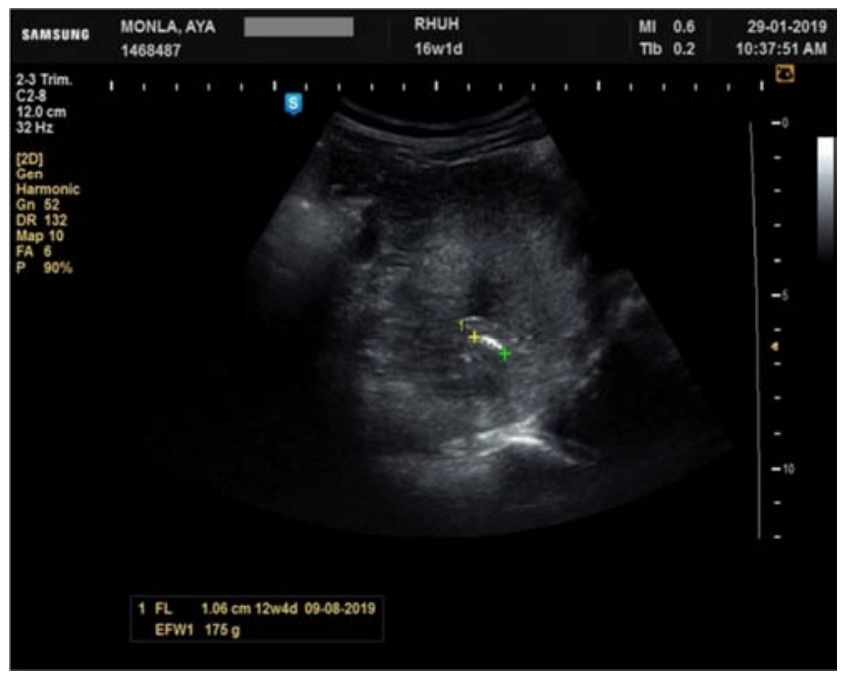

Figure 10. Measurement of the fetal femur - The distance between calipers.

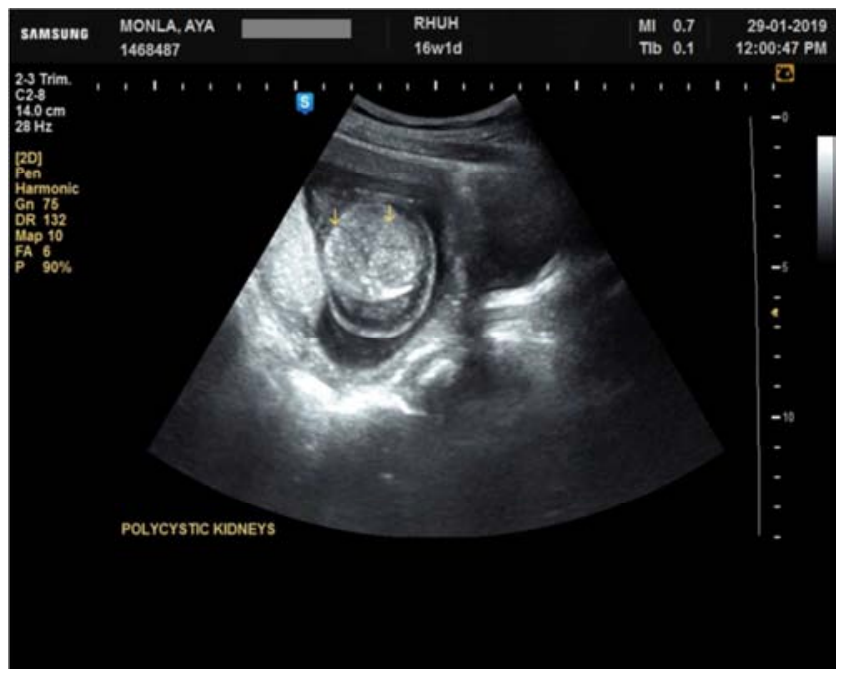

Figure 11. Axial image of the fetal abdomen showing large echogenic kidneys (arrows).

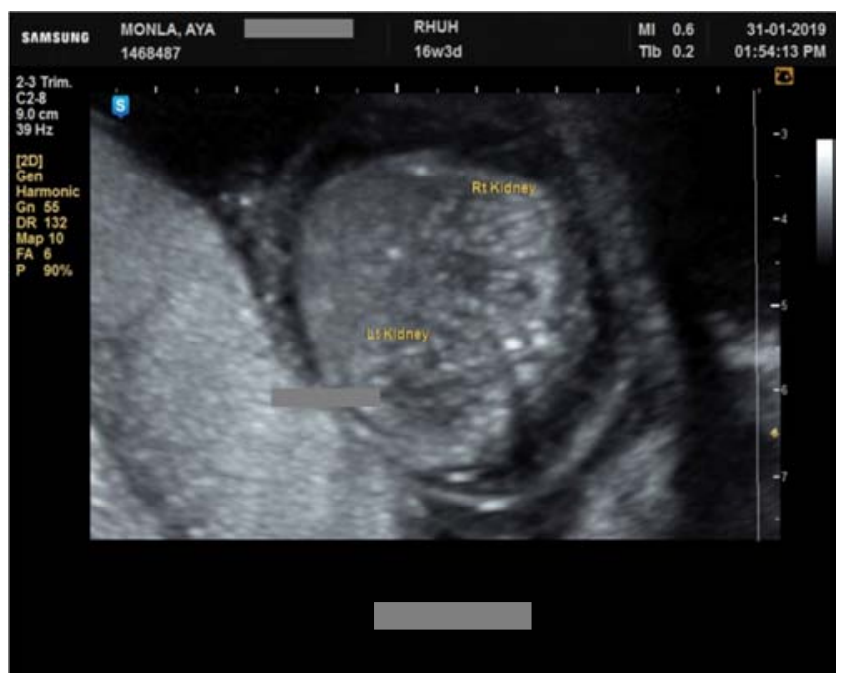

Figure 12. Transverse view of fetal abdomen: Diffuse involvement of both kidney. 


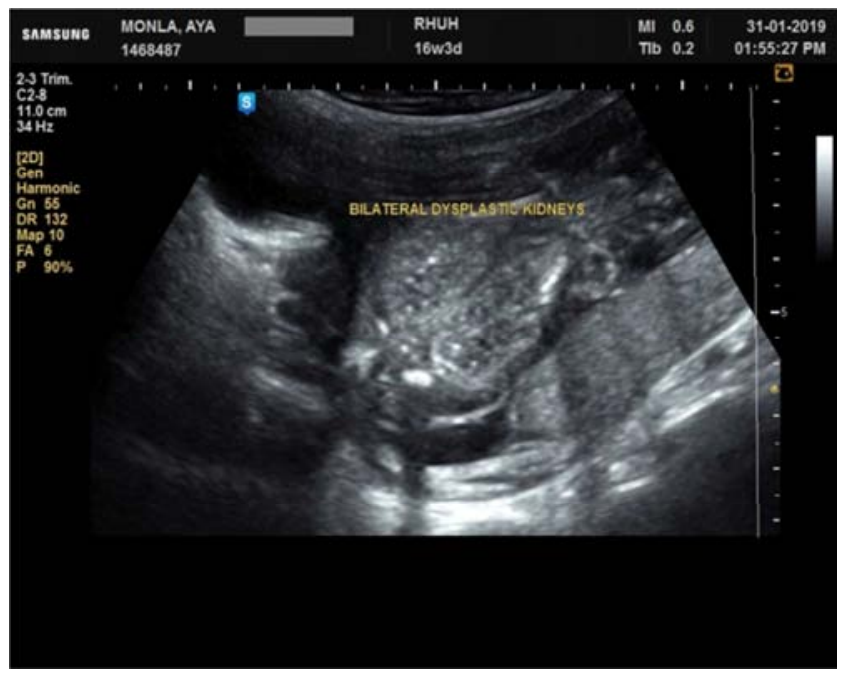

Figure 13. Medullary and cortical cysts. There are multiple diffuse cystic lesions. Bladder not visualized.

\section{Discussion}

In our case, despite the fact that there was neither a previous pregnancy nor a family history that could have guided the scanning, our patient had the opportunity, to be diagnosed antenatally with the modern ultrasound techniques. This is extremely important in such an inherited disease [1]. However, the diagnosis is considered late and the ultrasound exam should have been done earlier in the first trimester of pregnancy. If we consider the nationality of this patient, It was not reported an increased incidence in the Syrians [6].

The prenatal ultrasound that we performed in this case is considered to be in our days the best method available to diagnose MGS. Note that at the time of our ultrasonographic examination, only 2-dimensional (2D) and 3-dimensional (3D) modalities were available. However, the 4-dimensional modality (4D) would have been useful for us in order to assess not only the facial features and deformities but also the possible associated musculoskeletal malformations. MRI could have been also a valuable complement in assessing additional features or anomalies. However, the ultrasound findings were conclusive and sufficient enough to guide our treatment choices especially that the prognosis of this baby consists of a hundred percent mortality either in utero or shortly after birth. [7]

This syndrome is characterized by the triad of occipital encephalocele (60-80\%), cystic dysplasia of the kidneys (95$100 \%)$, postaxial polydactyly (75\%) and other variable congenital malformations [8-10]. The most characteristic feature of this triad is considered to be the Cystic dysplasia of the kidneys and this was clearly evident and reported in our case. Its detection always prompts the search for the other abnormal components of the MGS's triad. Although the occipital encephalocele of our case is the most common neural defect found in this condition, there are other neural reported associations like anencephaly, hydrocephalus and microcephaly. Furthermore, there is a wide variety of abnormalities that may be detectable sonographically with multiple combinations: oral clefting (either cleft lip or palate), genital anomalies (ambiguous genitalia), CNS malformations (Dandy-walker, Arnold-CHiari malformation, anophtalmia, microphtalmia), liver fibrosis (ductal plate malformation of the liver), cardiac lesions (ASD, coarctation of the aorta and pulmonary stenosis), and gastrointestinal anomalies like omphalocele. [11, 12, 13] In our case, we have two main ultrasound features that can be considered as the minimum diagnostic criteria: the encephalocele and the bilateral dysplastic kidneys. In fact, antenatal ultrasound examination can establish the correct diagnosis by identifying at least two of the described features. Note that due to the renal dysfunction, we were supposed to have oligohydramnios, the first sonographic finding that develops early in the second trimester in most cases. However, a normal amniotic fluid does not exclude the diagnosis of MGS. Therefore, our case is one of the Meckel Grubers that has a normal amniotic fluid despite the bladder absence. [14]

This evident posterior defect in the skull with protruding sac that contains brain tissue is the typical sonographic appearance of an encephalocele. If our patient did her first trimester ultrasound, this anomaly could have been diagnosed and provided more information about the CNS in comparison with the MRI [15]. The latter has superiority in comparison with ultrasound in the second and the third trimester and in the cases where there is confusion at the evaluation of the components of the sac. Diagnosing an encephalocele, on ultrasound can be either as an isolated lesion or a part of a syndrome: MGS and walker Warburg syndrome. The latter is characterized by lissencephaly, cerebellar hypoplasia, dandywalker cyst and ocular abnormalities [16] which is not our case. Four differential diagnosescan be evoked in addition to encephalocele in the presence of this occipital herniation on the antenatal ultrasound: midline dermoid cyst, hemangioma, vascular malformation of the scalp, and cystic hygroma. In our case the defect is covered by scalp. In the presence of distended hypoechoic sacs visualized on either side of the fetal neck and the subcutaneous fluid that extends the entire length of the fetus, we should evoke cystic hygroma as a differential diagnosis. However, cystic hygroma has an intact skull and spine on high-resolution transvaginal ultrasonography which is not the case here. In addition, the other differential diagnosis of vascular malformation of the scalp can be easily ruled out on color-flow mapping.

As for the kidneys, bilaterally dysplastic, enlarged hyperechogenic with medullary cysts, their reniform shape is not preserved. If we consider the classical classification of the congenital cystic renal disease, this kidney condition is a hereditary disorder taking part of a syndrome with multiple associated anomalies. Chaumoitre $\mathrm{K}$ et al in their study on 93 fetuses presenting with hyperechogenic kidneys found 9/93 with MGS and the other ones with the following syndromes: Ivemark II syndrome, trisomy 18, Jarcho-Levin syndrome, Beemer syndrome, ARPKD, ADPKD, Bardet-Biedl syndromeand Meckel-like syndrome.[17] Consequently, when bilateral dysplastic kidneys are revealed upon ultrasound examination, we should be thinking and searching 
of these syndromes despite some potential examination difficulties that might be present as bilateral renal disease is frequently associated with oligohydramnios. Trisomy 13 is considered as the main differential diagnosis to be confused with MGS since enlarged cystic kidneys are present in thirty percent of the cases and associated with neural tube defects. Despite the fact that the prognosis of MGS and that of trisomy 13 are the same, a karyotype is important in the presence of these findings because of the recurrence rate that is different: Trisomy 13 is a sporadic event; so determining the fetal microarray should be offered. Note that normal fetal kidneys can be physiologically hyperechogenic in the first and early second trimester, but the abnormality in this case is clearly evident based on the kidney size, Corticomedullary differentiation, level of renal echogenicity and location and size of renal cysts. We note that if this kidneys finding in our case was isolated, prenatal ultrasound alone should not be expected to predict etiology or long-term outcome in the absence of family history or postmortem or postnatal data.

\section{Conclusion}

Finally, if we consider the theory in such cases, the patient should be counseled regarding the termination of this pregnancy especially that MGS carry a poor prognosis and is not compatible with life. However, the termination of pregnancy in Lebanon raises complex, religious, social, legal and ethical issues to be checked with the hospital ethical committee.

On the other hand, the ultrasound in the context of genetic diseases, especially our MGS case, is considered as a secondary prevention tool. What outweight its screening potential is the preimplantation genetic diagnosis (PGD) at the DNA level, an important tool for primary prevention. [18] Due to the lack of facilities, this analysis cannot be done to the patient leading to a consequent missed opportunity to prevent the evident $25 \%$ recurrence risk of this disorder. However, it is very essential for the parents to have a full and extensive genetic counseling in order to help them with the required further management of future pregnancies.

\section{References}

[1] Mecke J. Passarge E Encephalocele, polycystic kidneys and polydactyly as an autosomal recessive trait simulating certain other disorders Ann Genet 1971; 14: 97.

[2] Karjalainen 0, Aula $P$, sepala $M$ et al Prenatal diagnosis of the Meckel Syndrome obstet Gynecol 1981; 47: 136.

[3] Gruber G B. Beitrage zur Frage "gekoppelter" Missbildungen akrocephalossyndactylie und dysencphalia splancnocystica. Beitr path Anat 1934; 93: 459-476.

[4] Meckel JF Beschreiburg zweier durch sehr ahnliche bildungsabweichungen entsteller Geschwister. Deutsch Archiv physiol 1822; 7: 99-172.

[5] Jha T, Bardhan J, Das B, Patra KK, Dhali B, Seth S. meckelGruber syndrome: a rare clinical entity J Indian Med Assoc 2010; 108 (9): 611-2

[6] Salonen R, Norio R, Reynolds James F. The Meckel syndrome: clinicopathological findings in 67 patients Am J Med Genet 1984. (4): 6771-689.

[7] Behairy NH, Talaat S, Saleem SN, El Raouf MA. magnetic resonance imaging in fetal anomalies: what does it add to 3D and 4DUS? Eur J Radiol 2009; 74 (1): 250-5.

[8] Nybergg DA, Hallesy D, Mahony BS et al Meckel Gruber syndrome: importance of prenatal diagnosisJ Ultrasound Med 1990 9: 691.

[9] Jones KL, Meckle Gruber syndrome Smith's recognizable pattern of human malformations $5^{\text {th }}$ ed. Philadelphia: W. B. Saunders; 1997.

[10] Celentano C, perfume F, Liberati M, Gallo G, Di Nisio Q, Rotmensch S, prenatal diagnosis of Meckel Gruber syndrome in a pregnancy obtained with ICSI, J Assist Reprod Genet 2006; 23 (6): 281-3.

[11] Wright C, Healicon R, English C, Burn J. Meckel syndrome: what are the minimum diagnostic criteria ? J Med Genet. 1994; 31 (6): 482-5.

[12] Salonen R, Norio $\mathrm{R}$ The Meckel syndrome in Finland: epidemiologic and genetic aspects. Am J Med Genet. 1984; 18 (4): 691-8.

[13] Shaheen R, Faqeih E, Seidahmed MZ, Sunker A, Alali FE, khadijah A et al A TCTN2 mutation defines a novel Meckel Gruber syndrome locus. Hum Mutat 201132 (6): 573-8.

[14] N Dahiya, S vijay, S Subhramaniam, Antenatal ultrasound diagnosis of Meckel Gruber SyndromeIndian J Radiol Imaging 2001; 11: 199-201.

[15] Monteagudo A, Timor-tritsch I fetal neurosonography of congenital brain anomalies. Ultrasonography of the prenatal and neonatal brain, McGraw-Hill, New York 2001.

[16] monteagudo A, Alayon A, Mayberry P, walker-warburg syndrome: case report and review of the literature, J ultrasound Med 2001; 20 (4): 419.

[17] Chaumoitre K, Brun M, Cassart M, Maugey-Laulom B, Eurin D, dider F, Avni EF, differential diagnosis of fetal hyperechogenic cystic kidneys unrelated to renal tract anomalies: A multicenter study, Ultrasound Obstet Gynecol 2006; 28 (7): 911.

[18] Brezina PR, Brezina DS, Kearns WG, preimplantation genetic testing BMJ 2012; 345: e5908. 